\title{
Domain game
}

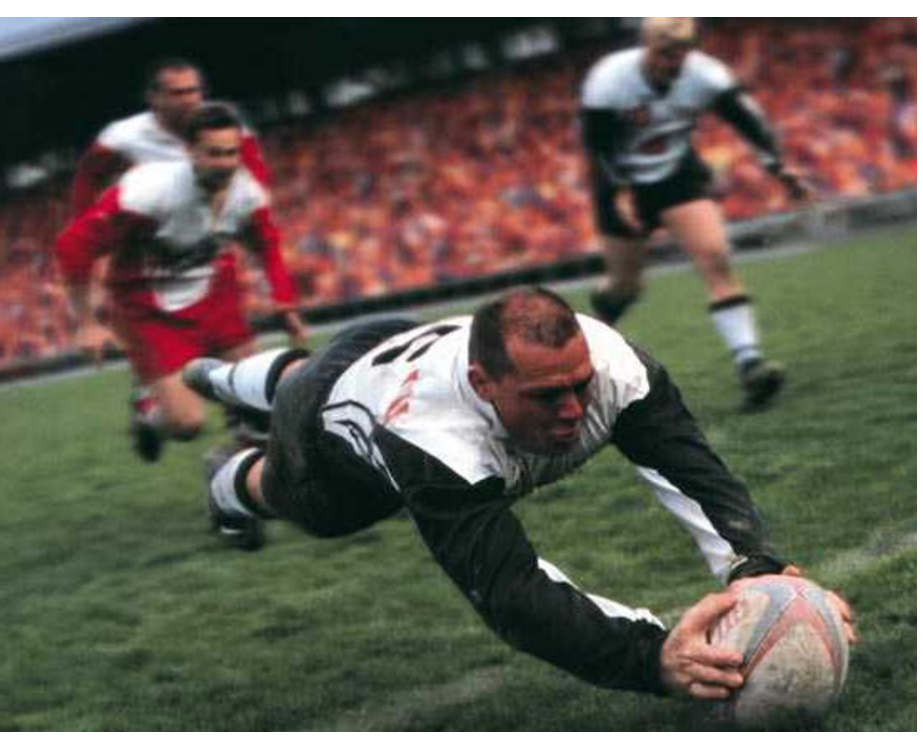

The inhibitor of apoptosis (IAP) family of proteins were identified first in baculoviruses and share a varying number of baculovirus IAP repeat domains (BIRs) and in some instances a RING finger domain with E3 ubiquitin (Ub) ligase activity. Pascal Meier and colleagues have identified another domain, a UBA domain, that confers oncogenic and survival properties to specific IAPs and might further explain their deregulated expression in human tumours.

Through the use of sequence analyses and structural prediction algorithms the authors found that IAPs containing three BIRs and a RING finger also contain a UBA domain. This domain, which is located between the third BIR and the RING finger, enables interaction with $\mathrm{Ub}$ and is found in proteins that recognize Ub-modified proteins and activate a variety of signalling pathways. Further experiments established that cIAP1 (also known as BIRC2) and Drosophila melanogaster IAP2 interact with both Lys48 and Lys63 poly(Ub) chains, whereas cIAP2 (BIRC3) and XIAP (BIRC4) interact only with Lys63 poly(Ub) chains. None of the IAPs tested interacted with monoubiquitylated residues. Protein domain deletion experiments indicate that these IAPs need to oligomerize before they can interact with poly(Ub) chains and that RING finger-mediated dimerization, but not E3 ligase activity, is required for this interaction.

So how does the interaction with poly(Ub) affect the function of IAPs? Deletion of cIAP1 results in sensitivity to tumour necrosis factormediated apoptosis. Re-expression of wild-type cIAP1 (cIAP1 $1^{\mathrm{WT}}$ ) in mouse embryonic fibroblasts (MEFs) that were null for the cIAP1 gene rescued this phenotype, whereas expression of cIAP1 with a mutated UBA domain $\left(\mathrm{CIAP} 1^{\mathrm{MF} / \mathrm{AA}}\right.$ ) did not. Amplification of $11 \mathrm{q} 22$, which contains the gene that encodes cIAP1, is often seen in human tumours, and overexpression of cIAP1 cooperates with either loss of $\mathrm{p} 53$ or deregulated expression of MYC during tumorigenesis. Unlike cells expressing MYC and cIAP $1^{\mathrm{WT}}$, co-expression of MYC and CIAP1 $1^{\mathrm{MF} / \mathrm{AA}}$ in liver cells failed to induce subcutaneous tumours when injected into immunocompromised mice.
cIAP2 is also involved in tumorigenesis as half of a reciprocal translocation with MALT1, resulting in MALT lymphoma. The cIAP2MALT1 fusion protein seems to lead to the activation of nuclear factor $\kappa \mathrm{B}$ $(\mathrm{NF \kappa B})$ through a mechanism that involves NEMO (inhibitor of NFKB kinase- $\gamma$ ). The BIR1 domain of cIAP2 is thought to be important in this process as it enables the oligomerization and therefore activation of cIAP2-MALT1. As 98\% of cIAP2MALT1 translocations retain the UBA domain, Meier and colleagues speculated that it might be crucial for tumorigenesis. Indeed, expression of a cIAP2 $2^{\mathrm{MF} / \mathrm{AA}}-\mathrm{MALT} 1$ mutant failed to activate NFאB in cell lines. Further analyses with this mutant showed that the UBA domain is required for cIAP2-MALT1 to stably associate with polyubiquitylated NEMO, but that CIAP2-MALT1 does not contribute to NEMO polyubiquitylation. Precisely how trapping of polyubiquitylated NEMO leads to NFאB activation remains to be elucidated.

So, IAPs not only suppress apoptosis but also bind polyubiquitylated proteins, a function which seems to be essential for their ability to promote survival, activate NFkB and act as oncogenes.

Nicola McCarthy

ORIGINAL RESEARCH PAPER Gyrd-Hansen, M. et al. IAPs contain an evolutionarily conserved ubiquitin-binding domain that regulates NF- $\mathrm{kB}$ as well as cell survival and oncogenesis. Nature Cell Biol. 19 Oct 2008 (doi 10.1038/ncb1789) 